\title{
ATIVIDADADES PEDAGÓGICAS INTERDISCIPLINARES: CIÊNCIA E CULTURA ARTICULADOS NA EDUCAÇÃO BÁSICA
}

\author{
Maria Aparecida de Carvalho ${ }^{1}$, Israel da Conceição Júnior ${ }^{2}$, \\ Marcia Regina de Souza Camanho ${ }^{3}$ \\ Universidade Federal do Espírito Santo \\ Centro de Educação - Campus Goiabeira \\ Av. Fernando Ferrari, 514 - Goiabeiras - Vitória - ES - CEP 29075-910.
}

\section{RESUMO}

Este trabalho tratou do estudo sobre projeto "A cultura por meio das ciências" realizado por professores da Escola Estadual de Ensino Fundamental e Médio "Marinete de Souza Lira", localizada no bairro Feu Rosa no município de Serra/ES, caracterizada por ser uma área de risco social. A ideia deste projeto, realizado entre junho a setembro de 2012, foi articular saberes como arte, filosofia, cultura, patrimônio, educação ambiental, química e o uso de equipamentos audiovisuais. A metodologia de trabalho envolve visitas pedagógicas, produção de relatórios, pesquisas bibliográficas e com imagens. Foram realizadas atividades de visitas pedagógicas com produção de relatórios, como na Universidade Federal do Espírito Santo e na Igreja dos Reis Magos, em Nova Almeida, Serra/ES. Este trabalho constituiu-se em uma atividade pedagógica interdisciplinar, promovendo o resgate da cultura local e o interesse dos alunos pelas ciências.

Palavras-chave: cultura e educação, interdisciplinaridade, patrimônio.

\section{ABSTRACT}

This work dealt with the study project "Culture through science " conducted by the State School Teachers of Elementary and Secondary Education "Marinete de Souza Lira", located in the neighborhood Feu Rosa in Serra of Espírito Santo State - Brazil, characterized by being an area of social risk. The idea of this project, conducted from June to September 2012, was articulate knowledge as art, philosophy, culture, heritage, environmental education, chemistry and the use of audiovisual equipment. The methodology involves educational visits, production of reports, literature searches and images. Activities of educational visits with production of reports, such as the Federal University of Espírito Santo and in the Church of the Magi were held in Nova Almeida, in Serra of Espírito Santo State - Brazil. This work consisted in an interdisciplinary pedagogical activity, promoting the recovery of local culture and students' interest in science.

Keywords: culture and education, interdisciplinarity, inheritance.

\footnotetext{
1 Professora de química. Mestre em Química. Rede Estadual da Educação Básica. Secretaria de Estado de Educação do Espírito Santo. E-mail: cida16ster@gmail.com

2 Professor de filosofia. Rede Estadual da Educação Básica. Secretaria de Estado de Educação do Espírito Santo. Email: idcjunior@gmail.com

3 Bacharel em design de produto. Rede Estadual da Educação Básica. Secretaria de Estado de Educação do Espírito Santo. E-mail: marcia_camanho@hotmail.com
} 


\section{Diversidade e Inclusão Social Interdisciplinaridade na Educação Básica}

\section{INTRODUÇÃO}

O texto dos PCNs de Ensino Médio, conforme ROSA (2007, p. 56) discute a possibilidade da interdisciplinaridade, como um dos eixos didático-metodológicos da proposta curricular, apostando na possibilidade de relacionar disciplinas por meio de atividades, de projetos de estudos, de pesquisa e ação. Dessa forma, o conceito de interdisciplinaridade é apresentado considerando:

[...] o fato trivial de que todo conhecimento mantém um diálogo permanente com outros conhecimentos, que pode ser de questionamento, de confirmação, de complementação, de negação, de ampliação, de iluminação de aspectos não distinguidos (Brasil, 1999, p.88).

Neste sentido, há uma dificuldade e/ou resistência dos professores em trabalhar a interdisciplinaridade. No que diz respeito a isso GATTÁS (2006, p. 24) vai afirmar:

A exigência interdisciplinar impõe às especialidades que transcendam suas próprias áreas, tomando consciência de seus limites e acolhendo as contribuições das outras disciplinas. Uma epistemologia de complementaridade, de convergência deve substituir a dissociação tão frequente na modernidade.

Hoje é marcante o fato de os educandos ficarem presos aos conteúdos que lhes são estabelecidos e que na maioria das vezes são tão extensos que o mesmo não consegue concluílos até o fim do ano letivo, e muitos professores não se sentem na obrigação da aplicação de um tema transversal, embora este seja de extrema importância. Mas:

A escola é um espaço privilegiado para estabelecer conexões e informações, como uma das possibilidades para criar condições e alternativas que estimulem os alunos a terem concepções e posturas cidadãs, cientes de suas responsabilidades e, principalmente, perceberem-se como integrantes do meio ambiente. A educação formal continua sendo um espaço importante para o desenvolvimento de valores e atitudes comprometidas com a sustentabilidade ecológica e social" (CUBA, 2012 apud LIMA, 2004). 


\section{Diversidade e Inclusão Social Interdisciplinaridade na Educação Básica}

Dessa forma, torna-se necessário que professores estejam abertos a essa discussão para que possam enxergar o compromisso de todos em relação a esse assunto, bem como perceber que é possível o trabalho interdisciplinar. Ressalta-se nesse ponto a importância de trabalhar a cultura local, visto que, com a globalização, evidencia-se que outra cultura se sobrepõe à cultura local, havendo uma desvalorização da cultura e história da localidade em que se vive. Informações de todo o mundo chegam muito rápido, há uma valorização daquilo que é externo, perdendo-se a consciência da história da própria região ou país. GATTÀS (2006, p. 324) faz uma afirmação que resume nossa posição a respeito da interdisciplinaridade:

A interdisciplinaridade é um processo que precisa ser vivido, reclama atitude interdisciplinar que se caracteriza por ousadia de busca, de pesquisa; transforma a insegurança num exercício de pensar, de construir; respeita o modo de ser de cada um e o caminho que cada um empreende na busca de autonomia; exige a elaboração de um projeto inicial que seja claro e coerente para que as pessoas sintam o desejo de fazer parte dele; pode ser aprendida e ensinada o que pressupõe o fato de perceber-se (sic) interdisciplinar.

A utilização da cultura como mediação interdisciplinar tornou-se oportuna, diante da diversidade dos campos que no ambiente escolar assumiam o desafio da integração, podendo abranger todas as áreas de conhecimento, diante da convicção que a realidade não é segmentada em disciplinas, mas integradas em vários campos tantos quantos possíveis. A afirmação da cultura como campo de "diáspora" (ROSA, 2007), onde os protagonistas utilizam o que sabem, mas sem medo do "perder-se", em uma atitude de aprendizagem autêntica e de amizade, onde a exploração do campo da cultura passa a ser um "ir além" dos conteúdos programáticos, fixos e um consequente abandono dessas formas, em prol do reconhecimento da interpelação dos vários fatores que formam a cultura de uma civilização e, consequentemente, o próprio indivíduo, e isso não significar abandonar a própria identidade e cair em uma situação ambígua (ROSA, 2007).

Se definirmos "cultura", segundo RIZZO (2008, p. 4), como tudo aquilo que o homem criou ou transformou, podemos chamar de "patrimônio cultural" o conjunto de todas as manifestações 


\section{Diversidade e Inclusão Social Interdisciplinaridade na Educação Básica}

de expressões tangíveis e intangíveis do ser humano. Como expressão material da atividade humana, os bens culturais podem ser de qualquer espécie. Desde que o homo habilis há cerca de dois milhões de anos atrás, começou a pegar elementos da natureza e trabalhá-los para conferir-lhes outra forma, como a pedra lascada, até os dias de hoje com a utilização de materiais sintéticos para fabricação de utensílios ou objetos de arte; assim todo e qualquer tipo de matéria intencionalmente trabalhada é considerada bem cultural material ou tangível. 0 patrimônio cultural material é no mínimo um documento histórico de uma época, podendo ser ainda, nos casos de obras de arte, o representante único de uma expressão artística. A importância de preservá-lo, portanto, é evidente: como documento histórico ele carrega informações sobre a trajetória do homem, seus hábitos, suas crenças, seus medos, a forma de interação com os outros e com a natureza; sua forma de subsistência e organização social, as rotas de comércio etc.

Conforme RIZZO (2005, p.8), a preservação ou conservação e restauração de bens culturais é uma atividade multi e interdisciplinar, e se situa numa interface que não apenas tange mas intercepta universos de outras atividades correlatas. 0 patrimônio cultural material é constituído por matéria, portanto, para preservá-lo é preciso conhecê-lo a nível atômico e molecular. É preciso conhecer a fundo as espécies com que ele foi feito e como elas se comportam, isto é: como elas reagem umas em relação às outras, por meio do tempo, com o meio ambiente, etc., em outras palavras, seus mecanismos de reação. É preciso conhecer ainda os produtos e os meios que se dispõe para atuar interferindo nestes processos, seja prevenindo, retardando ou restaurando os processos de deterioração.

As obras de arte, que fazem parte do patrimônio cultural da humanidade, estão sujeitas aos diferentes tipos de degradação: física, química e biológica. Do ponto de vista físico-químico, elas próprias são complexos sistemas em cujas interfaces (interna e com o ar) ocorrem inúmeras reações químicas. As obras estão sujeitas às alterações do meio ambiente, como variações de umidade e temperatura, assim como à adsorção de poluentes sob sua superfície e, ainda, possíveis interações sólido-sólido entre as espécies que compõe a obra, além, é claro, de atos de vandalismo. Do ponto de vista biológico, elas fazem parte do ecossistema e podem ser utilizadas como substratos por macro e microrganismos (RIZZO, 2008). Esse tipo de 


\section{Diversidade e Inclusão Social Interdisciplinaridade na Educação Básica}

desgaste pode ser recuperado com os serviços de restauração, feito pelo trabalho de pessoas de várias áreas, principalmente da química e das artes.

Tendo em vista os desafios ao diálogo das áreas do saber envolvidos nesse projeto que tem como ponto de contato a cultura, delimitamos como nossos objetivos: incentivar a valorização do patrimônio cultural capixaba por meio de um trabalho interdisciplinar entre química, arte e filosofia, bem como trabalhar a educação ambiental, visto que as obras de arte estão sujeitas aos diferentes tipos de degradação: física, química e biológica, sendo possível o estudo destes processos e o conhecimento dos patrimônios culturais a nível atômico e molecular por meio da química, e deste modo, incentivar mudanças no modo de olhar os patrimônios culturais e as ciências.

\section{PROCEDIMENTOS METODOLÓGICOS}

Primeiramente, o trabalho foi apresentado à comunidade escolar para que todos pudessem dar suas sugestões, ideias, contribuições e até mesmo buscar uma forma de se encaixar e trabalhar em conjunto, visto que o assunto é amplo e é possível uma junção de todas as áreas. Depois de apresentado e discutido o trabalho com a comunidade escolar decidiu-se trabalhar com os alunos do primeiro ano do ensino médio, a partir do trabalho teórico e com imagens, a história da ocupação do Espírito Santo, priorizando a região de Nova Almeida, onde se encontra a Igreja Reis Magos, patrimônio histórico da Serra, e onde foi realizado um trabalho pelo Núcleo de Restauração da UFES, abordando assim, ao mesmo tempo, os temas religião, arte e química, realizando visita guiada como forma de colocar em prática o que se estudou em sala de aula.

A primeira etapa do trabalho foi a associação entre química e arte, na explicação da importância do conhecimento das substâncias que compõe as obras de arte, e que é imprescindível para direcionar intervenções nos processos de restauração e conservação, além de fornecer informações sobre o período histórico da elaboração da obra, entendimento da técnica construtiva utilizada pelo artista e reconhecimento de falsificações (SERRATE, 2011), assim tratando da constituição atômica e molecular, além das formas de degradação dos patrimônios culturais, que podem ser físicas, químicas e biológicas (RIZZO, 2008). Neste 


\section{Diversidade e Inclusão Social Interdisciplinaridade na Educação Básica}

ponto, foi tratado com os alunos também acerca do vandalismo e a importância da conservação de sua história. Essa etapa consistiu em aulas teóricas expositivas com a utilização de textos e equipamentos audiovisuais sobre o assunto.

Preparados tecnicamente e teoricamente, os alunos fizeram uma visita ao Núcleo de Restauração, da UFES, sob a orientação de Gilca Flores e do responsável pelo mesmo, o artista Atílio Colnago. Essa visita foi realizada no dia 18/06/2012, tendo como primeiro momento uma palestra de apresentação sobre Ciência e Restauração e depois uma resumo sobre os trabalhos já realizados pelo Núcleo, além da visita ao laboratório onde os alunos tiveram uma explicação prática de tudo o que foi abordado.

Como próxima etapa, os alunos fizeram uma pesquisa sobre a história do estado do Espírito Santo e sobre os patrimônios culturais resultantes dessa história, além da explicação sobre os estilos artísticos encontrados nestes. Por meio da parceria com o projeto Visitar, do governo do estado, parte dos alunos fizeram uma visita pedagógica a vários dos principais patrimônios históricos do estado, e em outro momento, os demais alunos estarão fazendo a mesma visita. Como próximo passo, haverá a visita à Igreja Reis Magos no dia 25/07/2012, em que os alunos verão os resultados do trabalho de restauração feito no local pelo IPHAN e Núcleo de Restauração da UFES. Será também uma oportunidade dos alunos estabelecerem um contato direto com o fenômeno religioso na sua materialização espacial, por meio da ocupação do solo, das construções e objetos sagrados constatando o que ELIADE afirma (1992, p.21 e 22),

É fácil compreender porque o momento religioso implica o 'momento cosmogônico: o sagrado revela a realidade absoluta (grifo nosso) e, ao mesmo tempo, torna possível a orientação - portanto, funda o mundo, no sentido que fixa os limites e, assim, estabelece a ordem cósmica.

Essa percepção de como o homem religioso materializa esse relacionamento e percepção do sagrado espacialmente é importante para os alunos perceberem como isso se deu de forma concreta na nossa experiência, onde irão relacionar aquilo que estão estudando como "filosofia da religião" na disciplina de filosofia e com o projeto "diversidade religiosa" que está sendo executado juntamente com história. Durante essa visita os alunos, a partir do trabalho 


\section{Diversidade e Inclusão Social Interdisciplinaridade na Educação Básica}

teórico e com imagens que já vinha sendo desenvolvido em sala de aula, dialogam com os monitores, mas principalmente com a experiência concreta de centenas de anos, e a partir daí tiram as próprias conclusões. Esse contato que se estabelece entre alunos e patrimônio histórico-religioso tem por objetivo o reconhecimento por parte do aluno da rica contribuição histórica, ontológica e antropológica por parte da religião.

Além disso, os alunos estão fazendo uma pesquisa sobre pigmentos (composição química e processos de obtenção), e haverá aula prática sobre a obtenção de tintas, fazendo uma associação de Arte e os tipos de tintas utilizadas desde a Pré-História e por artistas conhecidos ao longo da História (SCHENKER, 2008). A finalização do trabalho será a escolha de uma linguagem artística para expressar suas ideias, pensamentos e/ou críticas de tudo o que foi trabalhado como forma de eternizar tal momento.

\section{RESULTADOS E DISCUSSÃO}

Na apresentação do projeto à comunidade escolar alguns professores aceitaram o desafio e a nova experiência imediatamente, enquanto outros se mostraram animados, tiveram acesso a todo um acervo de materiais como vídeos e textos tidos como base, mas não se manifestaram mais a respeito do assunto. Então aqueles professores que se prontificaram a trabalhar desde o início, nessa perspectiva, assumiram o projeto.

No andamento do projeto, os alunos produziram fotos e vídeos da visita ao Centro de Artes da UFES, ao Núcleo de Restauração, relacionando química e arte na obtenção, conservação e restauração de obras de arte. Nesta visita, os alunos também puderam estabelecer um contato direto com o fenômeno religioso por meio da percepção de como o homem religioso materializa esse relacionamento e percepção do sagrado espacialmente visto que grande parte dos patrimônios históricos culturais está relacionada com a religião e especialmente com as obras restauradas pelo Núcleo de Restauração, na igreja Reis Magos, em Nova Almeida, Serra-ES, no convento da Penha, com a imagem de Nossa Senhora da Penha onde foram demonstrados a especificidade do trabalho, a relação com a química e o respeito aos objetos enquanto sagrados de uma determinada comunidade religiosa, além da imagem do Cristo crucificado em Guarapari, que se encontrava no Núcleo de restauração da UFES, que foi um 


\section{Diversidade e Inclusão Social Interdisciplinaridade na Educação Básica}

exemplo de desrespeito aos objetos artístico-sagrados, onde o padre da paróquia engessou as articulações que ligavam antebraços ao tronco do Cristo que era utilizado como Morto, quando se lhe fechavam os braços no sábado de aleluia, que era uma tradição desde os jesuítas, e se lhe abria os braços crucificado no domingo de páscoa e durante o ano.

Os alunos manifestaram, durante as atividades e depois, uma melhor associação dos campos envolvidos diretos e mesmo os que não estavam envolvidos diretamente como disciplina, mas implícito nas atividades de pesquisa, como por exemplo, História. Outra observação a ser feita foi a demonstração de união na execução das atividades por parte dos alunos, sentimento que não se manifestava no início do ano, sendo de especial atenção em se tratando de uma escola em área de risco.

\section{CONSIDERAÇÕES FINAIS}

Percebeu-se que a resistência e/ou dificuldade dos professores em fazerem um trabalho interdisciplinar ainda é muito grande, visto que o projeto, aqui descrito, era muito mais abrangente e poderia incluir mais áreas de conhecimento. Isso ocorre principalmente devido à falta de entendimento ou mesmo de disposição para realização de tal trabalho. Mas não é um trabalho impossível, desde que o professor se abra para o novo e seja um professor pesquisador. Além disso, percebeu-se que é possível fazer um resgate da cultura local, além de promover o interesse dos alunos pelas ciências quando se trabalha desta forma, correlacionando o conteúdo de uma disciplina com o de outra, assim fazendo com que percebam a importância destas ciências e como estão presentes e influenciam na sua vida.

\section{AGRADECIMENTOS}

Ao Núcleo de Restauração da UFES, especialmente ao professor Atílio Colnago e Gilca Fores, à Prefeitura Municipal de Serra pela cessão do espaço da Igreja dos Reis Magos e EEEFM "Marinete de Souza Lira" pelo apoio e confiança.

\section{REFERÊNCIAS}


BRASIL, Ministério da Educação e do Desporto. Parâmetros Curriculares Nacionais para o Ensino Médio, Brasília: Secretaria de Ensino Médio, 1999.

CUBA, Marcos Antônio. Educação ambiental nas escolas. ECCOM, v. 1, n. 2, p. 23-31, 2010.

ELIADE, Mircea. 0 sagrado e o profano: A essência das religiões. São Paulo:

Martins Fontes, 1992.

GATTÁS, Maria Lúcia Borges; FUREGATO, Antonia Regina Ferreira. Interdisciplinaridade: uma contextualização. Acta Paul Enferm 2006; 19(3): 323-7.

RIZZO, Márcia de Mathias. Caracterização físico-química de materiais de esculturas de cera do Museu Alpino. Dissertação de mestrado. USP, 2008.

RIZZO, Márcia de Mathias. Como se dá o trabalho interdisciplinar. III Revista da APCRAssociação Paulista de Conservadores e Restauradores de Bens Culturais, v. III, p. 26-28, 2005.

ROSA, Maria Inês Petrucci. Experiências interdisciplinares e formação de professore (a)s de disciplinas escolares: imagens de um currículo diáspora. Revista Pró-posições, v.18, n. 2(53), p. 51-65, 2007.

SCHENKER, Libia. História da tinta através da arte ocidental. Revista Eletrônica Jovem Museologia, Vol. 3, n. 5, p. 60-97, 2008.

SERRATE, Júlia Wanguestel. Caracterização química de materiais pictóricos usados em escultura policromada- estudo de caso de uma escultura capixaba. Dissertação de mestrado, UFES, 2011. 